\title{
Sex-specific inhibition and stimulation of worker-reproductive transition in a termite
}

Qian Sun, Kenneth F. Haynes, Jordan D. Hampton, Xuguo Zhou*

Department of Entomology, University of Kentucky, Lexington, KY 40546-0091, USA

\section{*Corresponding Author:}

Dr. Xuguo "Joe" Zhou

Department of Entomology

University of Kentucky

S-225 Agricultural Science Centre North

Lexington, KY 40546-0091

Email: xuguozhou@uky.edu

Short Title: Termite worker-reproductive transition 


\section{Abstract}

2 In social insects, the postembryonic development of individuals exhibits strong phenotypic

3 plasticity in response to environment, thus generating the caste system. Different from eusocial

4 Hymenoptera, in which queens dominate reproduction and inhibit worker fertility, the primary

5 reproductive caste in termites (kings and queens) can be replaced by neotenic reproductives

6 derived from functionally sterile individuals. Feedback regulation of nestmate differentiation into

7 reproductives has been suggested, but the sex-specificity remains inconclusive. In the eastern

8 subterranean termite, Reticulitermes flavipes, we tested the hypothesis that neotenic

9 reproductives regulate worker-reproductive transition in a sex-specific manner. With this $R$.

10 flavipes system, we demonstrate a sex-specific regulatory mechanism with both inhibitory and

11 stimulatory functions. Neotenics inhibit workers of the same sex from differentiating into

12 additional reproductives, but stimulate workers of the opposite sex to undergo this transition.

13 Furthermore, this process is not affected by the presence of soldiers. Our results highlight the

14 extraordinary reproductive plasticity of termites in response to social cues, and provide insights

15 into the regulation of reproductive division of labour in a hemimetabolous social insect.

16

17 Keywords: caste differentiation, developmental plasticity, ergatoid reproductive, Reticulitermes

18 flavipes 


\section{Introduction}

20 Developmental plasticity plays an important role in the reproductive division of labour in social

21 insects (Page \& Amdam 2007). Caste differentiation in eusocial colonies is usually dependent on

22 social stimuli as well as other environmental cues (Hartfelder \& Engels 1998; Korb \& Hartfelder

23 2008). Although a fertilized egg is thought to be totipotent and able to develop into any caste,

24 only a few individuals eventually become reproductives. For example, female honeybee larvae

25 that are fed with royal jelly develop into queens, while others become workers (Kucharski et al.

26 2008). The presence of queens in social Hymenoptera also inhibits worker reproduction by

27 directly supressing their ovarian development, or through policing behaviour (Le Conte \& Hefetz

28 2008).

As with most social insects, termites have caste systems resulting from developmental

30 plasticity. In contrast to social Hymenoptera, hemimetabolous termites have both males and

31 females for all castes. Termite colonies are typically founded by a pair of dispersing alates,

32 which become the primary reproductives, i.e., kings and queens. In many "higher" termite genera

33 (Termitidae) and most "lower" termite genera (all other termite families), workers and nymphs

34 can differentiate into neotenic reproductives (ergatoids and nymphoids, respectively) and

35 reproduce in the natal colony (Myles 1999; Roisin 2000; Roisin \& Korb 2011). Neotenic

36 reproduction is implicated to play a critical role in the early evolution of termite eusociality

37 (Myles 1999). The fact that neotenics develop in response to orphaning (the absence of

38 reproductives) has led to the prevailing hypothesis that fertile reproductives would inhibit sexual

39 development (Long, Thorne \& Breisch 2003; Matsuura et al. 2010; Moore 1974; Noirot 1990). A

40 few studies, however, proposed the stimulatory effects of reproductive individuals on this

41 process. For example, in Mastotermes darwiniensis, neotenic reproductives were produced in the 
42 presence rather then the absence of neotenics, and female neotenics exhibited stronger

43 stimulatory activities on workers of both sexes than males (Watson, Metcalf \& Sewell 1975). In

44 Kalotermes flavicollis, the production of female neotenics was promoted by the presence of a

45 single male neotenic, while the stimulatory effect was not observed from female neotenics

46 (Lüscher 1964). Although kings and queens can both be replaced by neotenics, the sex-

47 specificity for either inhibition or potential stimulation is not conclusive in termites.

49 substantial economic and ecological importance (Su, Scheffrahn \& Cabrera 2001), is an ideal

50 system to study developmental plasticity. Reticulitermes workers have three morphologically,

51 behaviourally and functionally distinct developmental trajectories. They can undergo status quo

52 moults and remain as workers, differentiate into pre-soldiers followed by an additional moult into

53 soldiers, or develop into neotenic reproductives (i.e., ergatoids) (Lainé \& Wright 2003; Zhou, Oi \&

54 Scharf 2006). Our preliminary study in the eastern subterranean termite Reticulitermes flavipes

55 indicated that worker-reproductive transition was a lengthy process under orphaning condition

56 (30-90 days). If one of the reproductives (e.g., queen) is lost, a stimulatory function from the

57 remaining reproductive (e.g., king) that promotes the formation of neotenic reproductives of the

58 missing sex (e.g., female ergatoid) would be beneficial to the colony. We hypothesized that

59 worker-reproductive transition is regulated in a sex-specific manner in $R$. flavipes. Specifically,

60 reproductive individuals inhibit same-sex workers, but stimulate opposite-sex workers to

61 differentiate into ergatoids. To test this, we evaluated ergatoid formation in response to the

62 presence or absence of male or female reproductives. As soldier caste is present in all termite

63 species (Tian \& Zhou 2014), and previous studies suggest that soldiers potentially promote 
64 differentiation of reproductives (Watanabe et al. 2014), we also examined the effect of soldiers

65 on ergatoid formation.

67 Methods

68 Study System

69 Colonies of $R$. flavipes were collected from the Arboretum (Lexington, Kentucky, USA), and the

70 Red River Gorge area, Daniel Boone National Forest (Slade, Kentucky, USA). Colonies

71 consisted of workers, soldiers and nymphs at the time of collection. Freshly collected termites

72 were kept in Petri dishes and fed on moistened unbleached paper towel for one to two weeks.

73 Neotenic-headed colonies were obtained by transferring field collected termites to closed plastic

74 boxes $(45.7 \times 30.6 \times 15.2 \mathrm{~cm})$ filled with moistened wood mulch and pinewood blocks and

75 maintained for 6 months, when eggs and larvae appeared indicating the presence of fertile

76 neotenic reproductives. Primary-headed colonies were established by pairing female and male

77 sibling alates collected in Lexington, Kentucky, and they had been maintained in laboratory for 5

78 years by the time experiments started. All stock colonies and experimental termites were

79 maintained at $27 \pm 1^{\circ} \mathrm{C}$ in complete darkness.

\section{Bioassay to Test Sex-specific Regulation}

82 Fertile ergatoids of different sexes were used to test their influences on worker-reproductive

83 transition. These ergatoids were obtained from an orphaning assay, in which groups of 100

84 workers were kept in Petri dishes $(6.0 \mathrm{~cm}$ in diameter, $1.5 \mathrm{~cm}$ in height $)$ lined with moistened

85 unbleached paper towel for 60 days. Ergatoids that were actively reproducing (i.e., with eggs

86 present in dishes) were used in the subsequent assay. 
The same set-up was used to test how workers differentiate in response to ergatoids (Fig.

1). Groups of 100 workers were kept with: 1) no reproductives (F-M-); 2) one female ergatoid

$89(\mathrm{~F}+\mathrm{M}-) ; 3)$ one male ergatoid $(\mathrm{F}-\mathrm{M}+)$; or 4$)$ one pair of ergatoids $(\mathrm{F}+\mathrm{M}+)$. The ergatoids and

90 workers were from the same colony in each group. Fourteen replications using three colonies

91 were conducted, with one colony originally primary-headed and two colonies originally

92 neotenic-headed. Worker differentiation was observed daily for 60 consecutive days, and newly

93 formed ergatoids were removed and their sex was determined. Ergatoids were recognized by

94 slightly heavier cuticle pigmentation, elongated abdomen, and wider thorax than workers (Fig.

95 2). Female ergatoids were distinguished from males by their enlarged 7 th abdominal sternite. We

96 also removed eggs, newly formed pre-soldiers and any inter-caste (individuals with the degree of

97 mandible development intermediate between workers and pre-soldiers) every day to prevent their

98 potential influence on worker development.

99
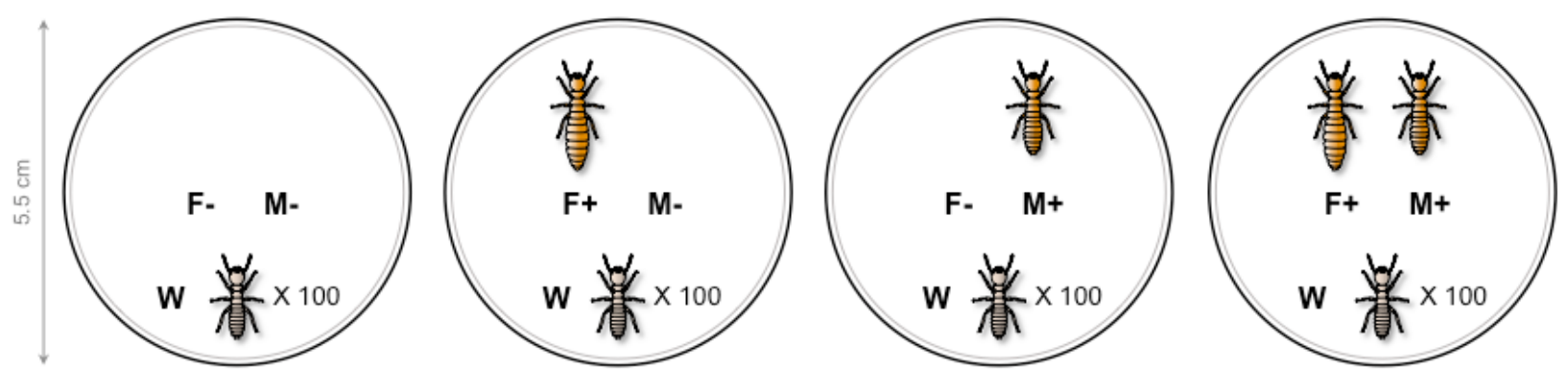

101 Figure 1. Experimental set-up. Each group of 100 workers were placed in a Petri dish lined with moistened

102 unbleached paper. Workers were kept with no reproductives (F-M-), one female ergatoid $(\mathrm{F}+\mathrm{M}-)$, one male ergatoid

$103(\mathrm{~F}-\mathrm{M}+)$, or one pair of ergatoids $(\mathrm{F}+\mathrm{M}+)$. Newly formed ergatoids, pre-soldiers, and eggs laid by reproductives were

104 removed every day for 60 consecutive days. FR: female ergatoid reproductive; MR: male ergatoid reproductive; W:

105 worker. Ergatoid reproductives used in the assay were actively reproducing, and female ergatoids were physogastric. 

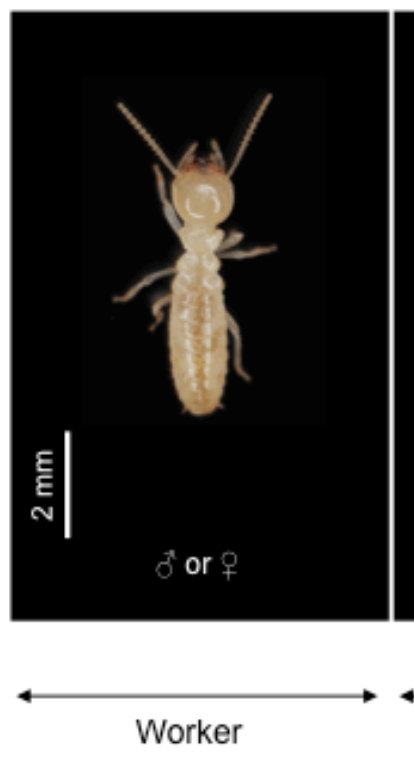

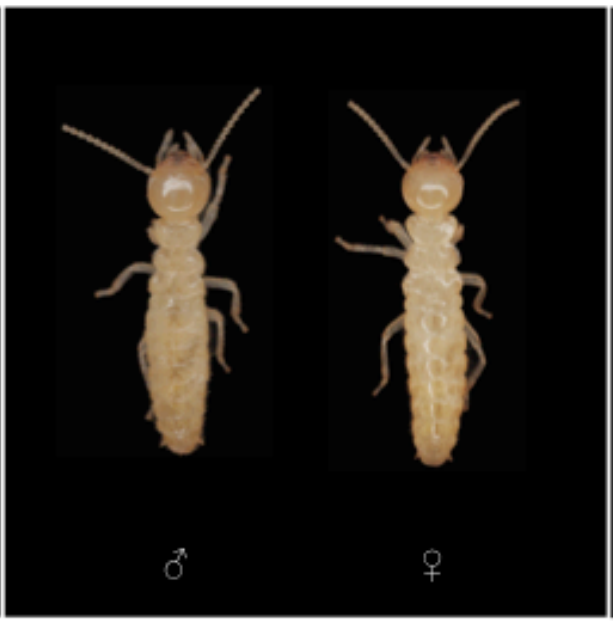

Newly differentiated

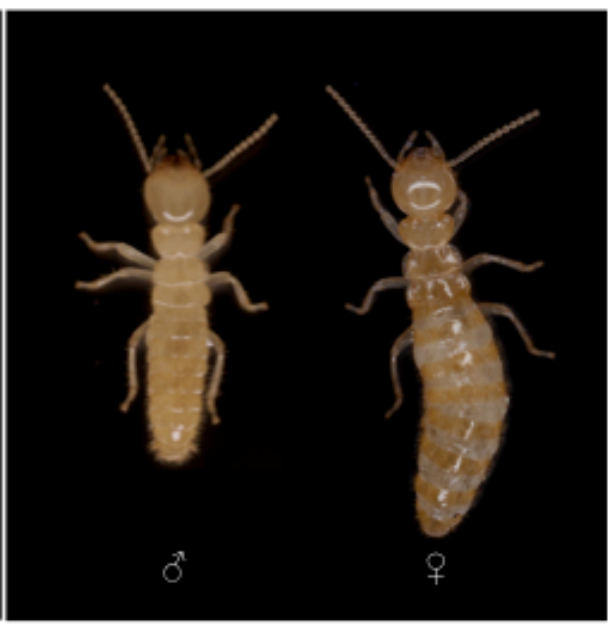

Actively reproducing

Ergatoid reproductives

107 Figure 2. Photographs of a worker, young ergatoids, and mature ergatoids. The young ergatoids were about 10

108 days post differentiation. The mature ergatoids were six months post differentiation and actively reproducing.

\section{Bioassays to Test Soldier Effect}

111 Two orphaning assays were conducted to test influence of soldier caste on ergatoid

112 differentiation. The first assay (“single-soldier orphaning assay”) simulated natural orphaning

113 condition where freshly collected workers were separated into groups of 100 individuals, and

114 each group was placed with one soldier (Soldier+) or no soldier (Soldier-). Termites were

115 maintained in Petri dishes $(6.0 \mathrm{~cm}$ in diameter, $1.5 \mathrm{~cm}$ in height) lined with moistened

116 unbleached paper towel as food source. Termites were allowed to undergo changes in caste

117 differentiation in the dishes without disturbance, and caste composition and mortality of each

118 group was documented at the end of 60 days. A total of 42 and 45 replicates from four colonies

119 were conducted for soldier+ and soldier- treatment, respectively.

120 We further performed the second assay ("multiple-soldier orphaning assay"), which was

121 conducted with an increased soldier stimulus and observed daily for 60 consecutive days. Groups 
122 of 100 workers were isolated from neotenic-headed colonies, and they were companioned with

123 either four soldiers (Soldier++) or no soldier (Soldier-). Newly differentiated individuals

124 (ergatoids and pre-soldiers) were removed to prevent their potential influence on worker

125 development, and an equal number of workers to the removed individuals were added to the

126 group to keep group size consistent. Termites were maintained in Petri dishes $(3.5 \mathrm{~cm}$ in

127 diameter, $1.5 \mathrm{~cm}$ in height) provided with moistened unbleached paper towel. A total of 10

128 replicates from two colonies were conducted for both soldier++ and soldier- treatments.

130 Data Analyses

131 Data were analysed using Statistix 10 (Analytical Software, Tallahassee, FL, USA). In the assay

132 that tested sex-specific regulation, Wilcoxon rank-sum test was performed on the cumulative

133 numbers ergatoids between each treatment and the control on every assay day. In both single-

134 and multiple-soldier orphaning assays, unpaired $t$-test was performed on numbers of

135 differentiated individuals and mortality. To obtain values that fit the assumptions of parametric

136 test, data were transformed through square root $(\mathrm{x}+0.5)$ on the combined numbers of pre-

137 soldiers and soldiers in the single-soldier orphaning assay, and numbers of female and male

138 ergatoids in the multiple-soldier orphaning assay. Because the pattern of regulation was

139 consistent in all colonies, results were pooled for statistical analyses.

\section{Results}

\section{Ergatoid Formation in Response to Reproductives of Different sexes}

143 Under orphaning condition (F-M-), $4.1 \pm 1.3 \%$ and $2.5 \pm 0.7 \%$ of workers differentiated into

144 female and male ergatoids, respectively, in 60 days (mean \pm SEM, Fig. 3). The presence of a 
145 single female $(\mathrm{F}+\mathrm{M}-)$ or a pair of ergatoids $(\mathrm{F}+\mathrm{M}+)$ significantly inhibited the formation of

146 additional female ergatoids $(0.2 \pm 0.2 \%$ and $0.1 \pm 0.1 \%$, respectively); however, the presence of

147 a single male ergatoid (F-M+) significantly stimulated the formation of female ergatoid (26.8 \pm

148 3.0\%) (Fig. 3(a); $P<0.01$, Wilcoxon rank-sum test, one-sided, $\mathrm{n}=14$ ). Similarly, significantly

149 fewer male ergatoids differentiated in the presence of a single male $(\mathrm{F}-\mathrm{M}+)$ or a pair of ergatoids

$150(\mathrm{~F}+\mathrm{M}+)(0 \%$ and $0.1 \pm 0.1 \%$, respectively $)$, while significantly more of them formed when a

151 single female ergatoid was present (F+M-) $(14.3 \pm 1.6 \%)$ (Fig. 3(b); $P<0.01$, Wilcoxon rank-

152 sum test, one-sided, $\mathrm{n}=14)$.

153 Within 60 days, female and male ergatoids differentiated in 10 and 9 replicates,

154 respectively, out of 14 total replicates under the orphaning condition (F-M-). The formation of

155 ergatoids in all 14 replicates was stimulated by a single ergatoid of the opposite sex. Under this

156 stimulation, developmental time for the first ergatoid was significantly reduced (female: $19 \pm 3.3$

157 days, $\mathrm{n}=14$ in F-M+, compared with $38 \pm 3.7$ days, $\mathrm{n}=10$ in F-M-; male: $21 \pm 1.8$ days, $\mathrm{n}=14$

158 in F+M-, compared with $35 \pm 5.4$ days, $\mathrm{n}=9$ in F-M-; Wilcoxon rank-sum test, one-sided, $P<$

159 0.01). There was no significant difference on mortality within 60-day assay period among

160 treatments (Fig. 4; ANOVA, $F_{3,52}=1.5, P>0.05 ; \mathrm{n}=14$ ). 


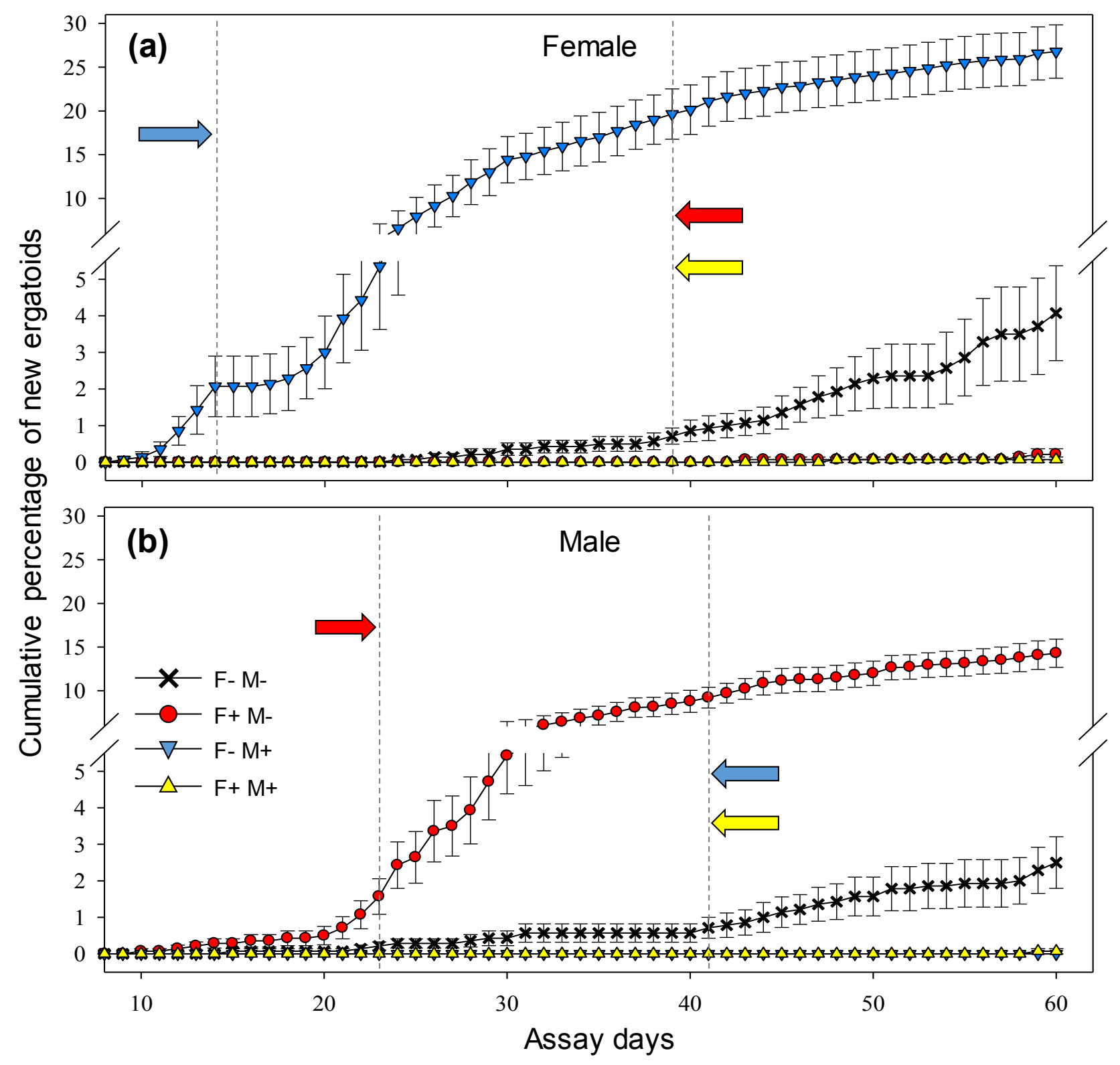

162 Figure 3. Ergatoid formation in response to fertile ergatoids of different sexes. Cumulative percentage of newly

163 differentiated female (a) and male (b) ergatoids is shown (mean \pm SEM). Stimulation (forward arrows) and

164 inhibition (reverse arrows) refer to significantly more and fewer ergatoids formed, respectively, relative to control

165 (F-M-), and dash line next to the tip of arrow indicates the day when the significant difference started (Wilcoxon

166 rank-sum test, one-sided, $P<0.01 ; \mathrm{n}=14$ for all treatments). Symbols and arrows of the same colour correspond to

167 the same treatment. 

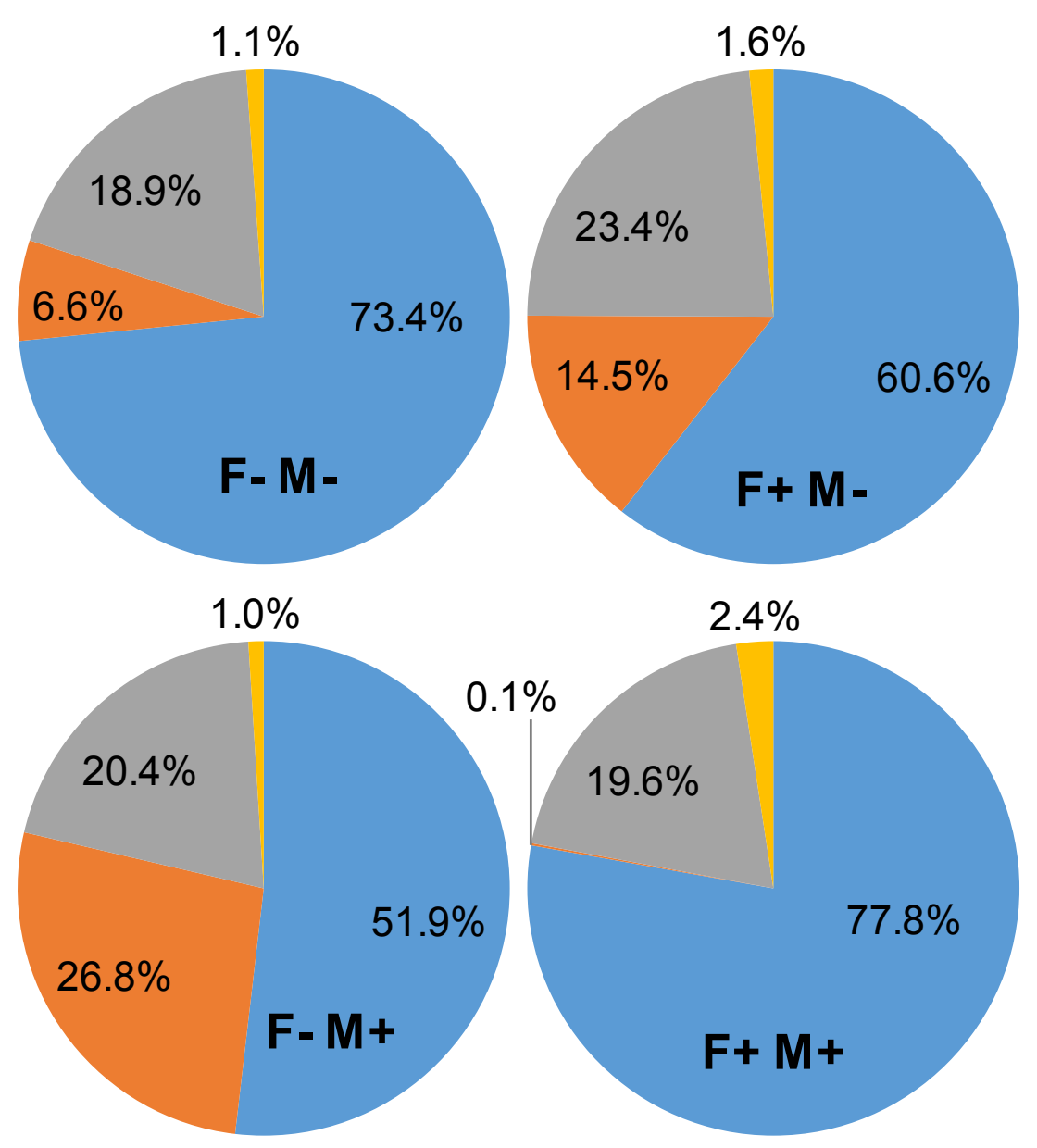

170 Figure 4. Developmental endpoint of workers in 60 days. "Others" includes pre-soldiers and inter-castes, and the

171 latter refers to individuals with the degree of mandible development between workers and pre-soldiers after

172 moulting. There was no significant effect of treatment on mortality (ANOVA, $F_{3,52}=1.5, P>0.05 ; \mathrm{n}=14$ pooled

173 from three colonies).

\section{4}

\section{Ergatoid Formation in Response to Soldier Caste}

176 In both assays, ergatoids were differentiated from workers in response to orphaning condition,

177 and there were no significant effects of soldiers on ergatoid formation (Fig. 5). In the single-

178 soldier orphaning assay, soldier caste did not significantly influence the number of female or 
179 male ergatoids in 60 days (Fig. 5(a); female: $t_{85}=1.64, P>0.05$; male: $t_{85}=1.64, P>0.05$;

180 unpaired $t$-test, two-sided, $\mathrm{n}=42$ for Soldier+; $\mathrm{n}=45$ for Soldier-). At the end of the assay,

181 mortality between Soldier+ and Soldier- groups were not significantly different (Fig. 5(b); $t_{42}=$

$1820.67, P>0.05$; unpaired $t$-test, two-sided, $\mathrm{n}=22$ randomly selected from both soldier + and

183 soldier- groups). The presence of one soldier significantly supressed the differentiation of pre-

184 soldiers and soldiers, and the increased total number of pre-soldiers and soldiers are $0.43 \pm 0.10$

185 in Soldier+ groups and $0.91 \pm 0.14$ in Soldier- groups $\left(t_{85}=2.71, P<0.01\right.$; unpaired $t$-test, two-

186 sided; data were transformed (square $\operatorname{root}(\mathrm{x}+0.5)) ; \mathrm{n}=42$ for Soldier,$+ \mathrm{n}=45$ for Soldier-).

187 Similarly, in the multiple-soldier orphaning assay, the soldier caste did not significantly

188 influence the accumulative number of female or male ergatoids in 60 days (Fig. 5(c); female: $t_{18}$

$189=0.57, P>0.05$; male: $t_{18}=0.44, P>0.05$; unpaired $t$-test, two-sided; data were transformed

190 (square root $(\mathrm{x}+0.5)) ; \mathrm{n}=10$ for both Soldier++ and Soldier- groups). Mortality was not

191 significantly influenced by the presence of 4 soldiers (Fig. $5(\mathrm{~d}) ; t_{18}=1.02, P>0.05$; unpaired $t$ -

192 test, two-sided, $\mathrm{n}=10$ for both Soldier++ and Soldier- groups). 

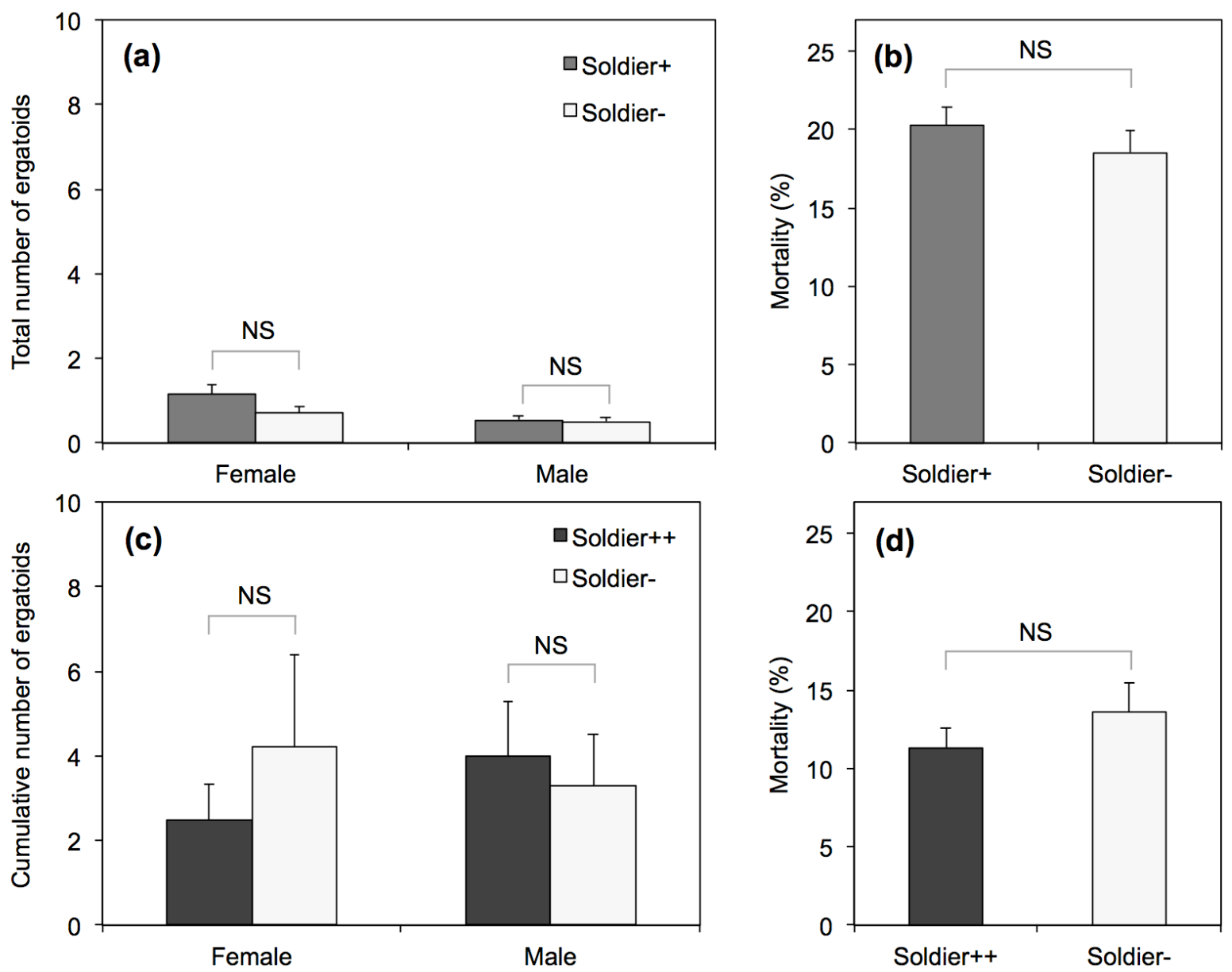

195 Figure 5. Soldier impacts on ergatoid formation. In a single-solider orphaning assay, total numbers of female and male ergatoids presented (mean \pm SEM) (a) and mortality $(\%$, mean \pm SEM) (b) between soldier present and absent

197 groups in 60 days are shown. In a multiple-soldier orphaning assay, cumulative numbers of female and male

198 ergatoids differentiated (mean \pm SEM) (c) and mortality (\%, mean \pm SEM) (d) between soldier present and absent

199 groups in 60 days are shown. In the single-soldier assay, newly differentiated ergatoids were left in groups;

200 Soldier+: each group consisted of 100 workers and one soldier at the start of assay; Soldier-: each group consisted of

201100 workers only; NS: no significant difference (unpaired $t$-test, two-sided, $P>0.05 ; \mathrm{n}=42$ for Soldier,$+ \mathrm{n}=45$ for

202 Soldier-). In the multiple-soldier assay, newly differentiated ergatoids were removed and replaced with workers

203 every day; Soldier++: each group consisted of 100 workers and four soldiers at the start of assay; Soldier-: each

204 group consisted of 100 workers only; NS: no significant difference (unpaired $t$-test, two-sided, $P>0.05 ; \mathrm{n}=10$ for

205 both Soldier++ and Soldier-). 


\section{Discussion}

208 These results support our hypothesis that regulation of worker-reproductive transition by fertile

209 reproductives is sex-specific. More importantly, our empirical evidence demonstrated that the

210 dual regulation (inhibition and stimulation) is employed by both sexes. Ergatoid differentiation

211 occurs after more than 30 days in response to orphaning, but can be significantly accelerated by

212 the presence of a potential mate. Such stimulation by the opposite sex benefits the colony by

213 enabling it to resume reproduction soon after the loss of a queen or a king. Inhibition of

214 development by the same sex, on the other hand, prevents unnecessary investments in

215 reproduction, which, in turn, would be a loss in the labour force. This sex-specific regulation

216 suggests that the development of reproductives is strictly dependent on the reproductive needs of

217 the colony.

218 Neotenic reproduction is common in termites; however, regulation of neotenic

219 differentiation varies among species (Grassé \& Noirot 1960; Lüscher 1964; Matsuura et al.,

220 2010; Miyaguni, Sugio \& Tsuji 2013; Watson, Metcalf \& Sewell 1975). In R. speratus, female

221 reproductives inhibit the differentiation of female neotenics, but do not influence the formation

222 of male neotenics (Matsuura et al. 2010). Compared with $R$. flavipes, female ergatoid formation

223 is faster in R. speratus in response to orphaning, and the formation of nymphoids are faster than

224 ergatoids in R. speratus (Matsuura et al. 2010; Miyata, Furuichi \& Kitade 2004). Orphaning

225 assays have also been conducted in other congeneric species including R. grassei (Pichon et al.

226 2007) and R. urbis (Ghesini \& Marini 2009), which confirmed the inhibitory effect of

227 reproductive pairs, but the regulation by each sex remains unclear. Stimulation by neotenic

228 reproductives has been suggested previously in primitive termite species. In $K$. flavicollis, the

229 formation of female neotenics was stimulated by the extracts of male reproductives (Lüscher 
230 1964). In M. darwiniensis, formation of neotenics was promoted in the presence rather than the

231 absence of other neotenics. Although sex-specificity was not confirmed, female neotenics

232 exhibited stronger stimulatory effects than males and the pair (Watson \& Abbey 1985; Watson,

233 Metcalf \& Sewell 1975).

234 In comparison, $R$. flavipes neotenics exhibit sex-specific inhibition and stimulation in

235 both sexes (Fig. 6a). Such a regulatory pattern consists of all possible directions of social

236 regulation, therefore presents a model for understanding the pheromonal and developmental

237 mechanisms underlying neotenic reproduction. Given that previous studies on the differentiation

238 of neotenic reproductives were incomplete or inconclusive (Fig. 6b-6d), this study also provides

239 an opportunity for us to re-examine the sex-specificity hypothesis across termite taxa. Foraging

240 populations of $R$. flavipes contain about $2 \%$ or less soldiers (Haverty \& Howard 1981; Howard \&

241 Haverty 1980), while higher proportions (close to 4\%) were observed in nest areas where

242 neotenics are present (Howard \& Haverty 1980). Soldiers were considered to induce the

243 differentiation of reproductives (Tian \& Zhou 2014; Watanabe et al. 2014), however, our results

244 indicated that soldier caste does not play a significant role in regulating ergatoid formation in $R$.

245 flavipes. It is worth noting that if ergatoids were not removed from the groups (single-soldier

246 orphaning assay), the total number of ergatoids was lower than the cumulative number of

247 ergatoids if they were constantly removed (multiple-soldier orphaning assay) within the same

248 period of time. Although the two assays were conducted separately and not entirely comparable,

249 this result could be explained by newly formed ergatoids suppressing formation of additional

250 ergatoids through pheromones or policing behaviour. 

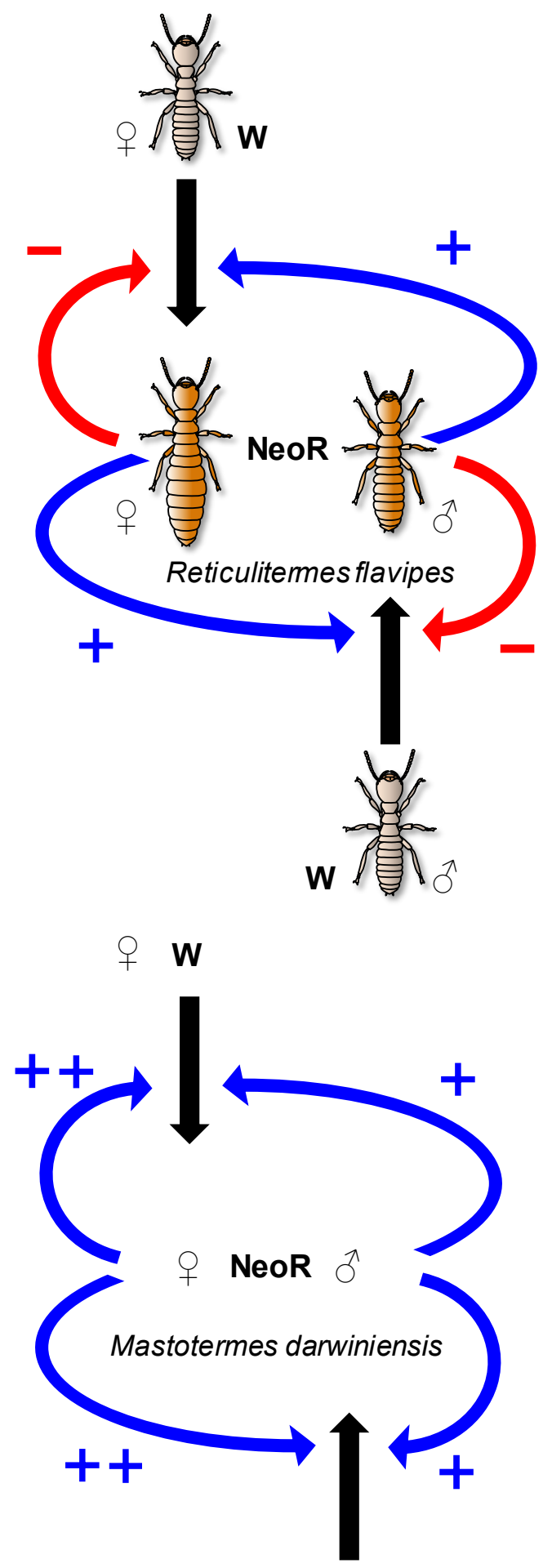

w え
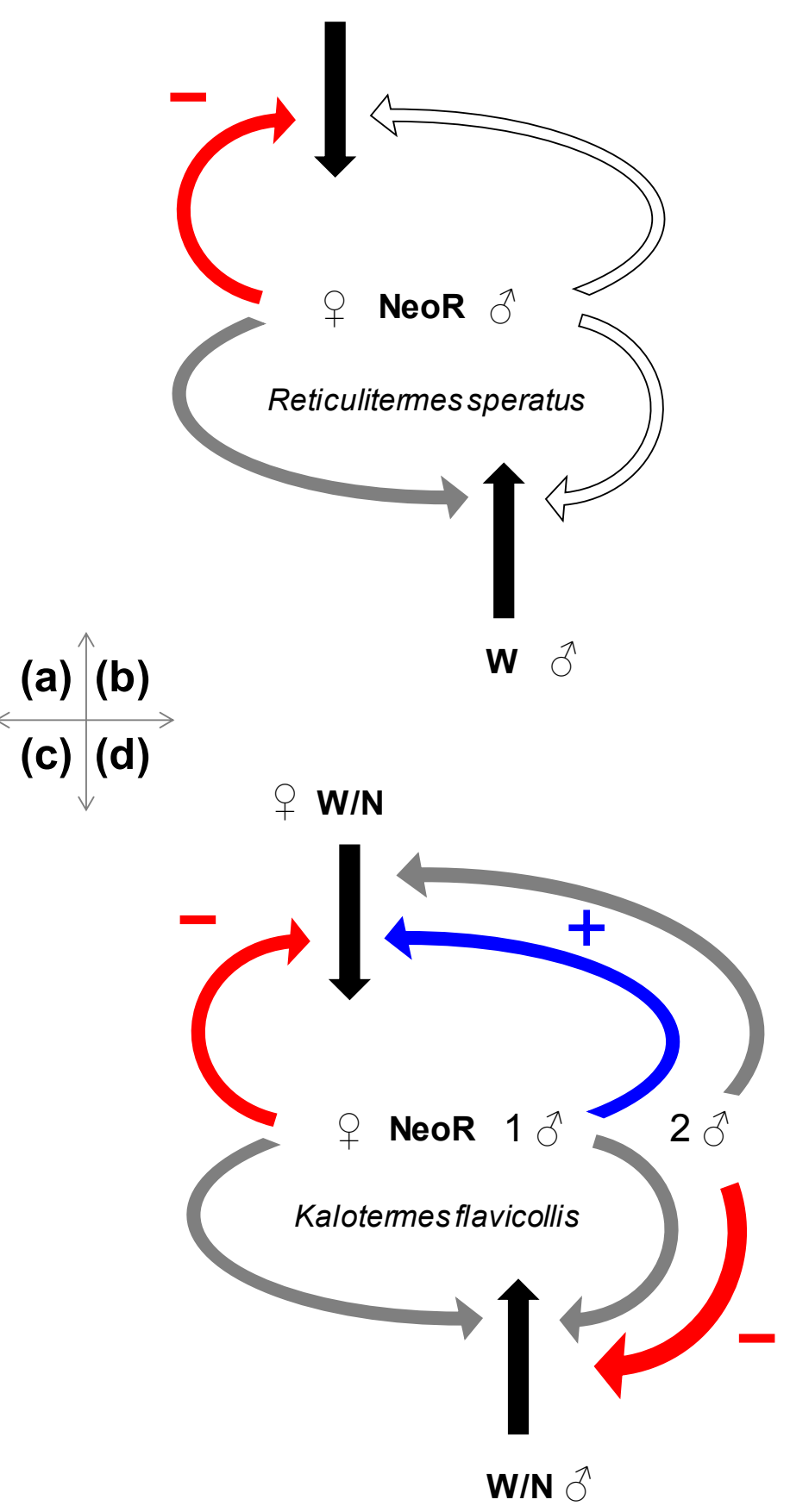
253 Figure 6. Comparison of feedback regulation of neotenic differentiation in four termites. (a) Sex-specific

254 inhibition and stimulation are demonstrated for both females and males in R. flavipes (this study). (b) In R. speratus,

255 female neotenics inhibit differentiation of females, but does not influence males; the effects of male neotenics were

256 not reported. (c) In M. darwiniensis, both female and male neotenics stimulate neotenic differentiation, but not in a

257 sex-specific manner; females exhibit stronger stimulation. (d) In K. flavicollis, female neotenics inhibit

258 differentiation of females, but the effect of males depends on number. One male neotenic shows opposite-sex

259 stimulation, while two males exhibit same-sex inhibition. W: worker; N: nymph; NeoR: neotenic reproductive.

262 differentiation in response to social cues. The results from this study add a new dimension to the

263 prevailing view that reproductives inhibit worker-reproductive transition in termites (Noirot

264 1990). Much remains to be investigated about the regulatory mechanisms of caste differentiation,

265 including the identification of inhibitory and stimulatory pheromones from reproductives. The

266 active substances or blends must be sex-specific. The search of reproductive pheromones in

267 termites should include volatile compounds (Matsuura et al. 2010), cuticular hydrocarbons

268 (Liebig, Eliyahu \& Brent 2009) as often observed in Hymenoptera (Van Oystaeyen et al. 2014),

269 and proteinaceous secretions (Hanus et al. 2010). The sex-specificity and the dual effect of

270 reproductive cues reflect unique adaptation and regulation of caste differentiation in

271 hemimetabolous termites. 


\section{Acknowledgments}

273 We thank Dr. Li Tian (Pennsylvania State University) for his help with photography, and

274 members of the Zhou lab for their comments and discussion. This study was supported by

275 William L. and Ruth D. Nutting Student Research Grant from the International Union for the

276 Study of Social Insects (North American Section), Kentucky Opportunity Fellowship from the

277 University of Kentucky to Q.S., and the USDA National Institute of Food and Agriculture Hatch

278 project (Accession Number: 1004654) to X.Z. Any opinions, findings, conclusions, or

279 recommendations expressed in this publication are those of the author(s) and do not necessarily

280 reflect the view of the National Institute of Food and Agriculture (NIFA) or the United States

281 Department of Agriculture (USDA). This is publication No. 17-08-002 of the Kentucky

282 Agricultural Experiment Station and is published with the approval of the Director. The granting

283 agencies have no role in the study design, data collection and analysis, decision to publish, or

284 preparation of the manuscript.

\section{Author Contributions}

287 Q.S., K.F.H. and X.Z. designed the experiments, Q.S. and J.D.H conducted the experiments,

288 Q.S. and K.F.H analysed the data, Q.S. drafted the manuscript, and K.F.H and X.Z. revised the

289 manuscript. All authors approved the final manuscript. 


\section{References}

293 Ghesini, S. \& Marini, M. (2009) Caste differentiation and growth of laboratory colonies of Reticulitermes urbis (Isoptera, Rhinotermitidae). Insectes Sociaux, 56, 309-318. doi: $10.1007 / \mathrm{s} 00040-009-0025-1$

Grassé, P. P. \& Noirot, C. (1960) Role respectif des males et des femelles dans la formation des sexués néoténiques chez Calotermes flavicollis. Insectes Sociaux, 7,109-123. doi:

Hanus, R., Vrkoslav, V., Hrdý, I., Cvačka, J. \& Šobotník, J. (2010) Beyond cuticular hydrocarbons: evidence of proteinaceous secretion specific to termite kings and queens. Proceedings of the Royal Society of London B: Biological Sciences, 277, 995-1002. doi: $10.1098 /$ rspb.2009.1857

303 Hartfelder, K. \& Engels, W. (1998) Social insect polymorphism: hormonal regulation of

307 Haverty, M. \& Howard, R. (1981) Production of soldiers and maintenance of soldier proportions San Diego.

311 Howard, R.W. \& Haverty, M.I. (1980) Reproductives in mature colonies of Reticulitermes

312 flavipes: abundance, sex-ratio, and association with soldiers. Environmental Entomology, 
314 Korb, J. \& Hartfelder, K. (2008) Life history and development - a framework for understanding

315 developmental plasticity in lower termites. Biological Reviews, 83, 295-313. doi: 10.1111/j.1469-185X.2008.00044.X

317 Kucharski, R., Maleszka, J., Foret, S. \& Maleszka, R. (2008) Nutritional control of reproductive status in honeybees via DNA methylation. Science, 319, 1827-1830. doi:

Lainé, L.V. \& Wright, D.J. 2003. The life cycle of Reticulitermes spp. (Isoptera: Rhinotermitidae): what do we know? Bulletin of Entomological Research, 93, 267-278.

323 Le Conte, Y.\& Hefetz. A. (2008) Primer pheromones in social Hymenoptera. Annual Review of Entomology, 53, 523-542. doi: 10.1146/annurev.ento.52.110405.091434 status in the termite Zootermopsis nevadensis. Behavioral Ecology and Sociobiology, 63,

Long, C.E., Thorne, B.L. \& Breisch N.L. (2003) Termite colony ontogeny: a long-term assessment of reproductive lifespan, caste ratios and colony size in Reticulitermes flavipes (Isoptera: Rhinotermitidae). Bulletin of Entomological Research, 93, 439-445. doi: https://doi.org/10.1079/BER2003258

332 Lüscher, M. (1964). Die spezifische Wirkung männlicher und weiblicher Ersatzgeschlechtstiere auf die Entstehung von Ersatzgeschlechtstieren bei der Termite Kalotermes flavicollis

335 Matsuura, K., Himuro, C., Yokoi, T., Yamamoto, Y., Vargo, E.L. \& Keller L. 2010.

336 Identification of a pheromone regulating caste differentiation in termites. Proceedings of 
the National Academy of Sciences of the United States of America, 107, 12963-12968. doi: $10.1073 /$ pnas. 1004675107

Miyaguni, Y., Sugio K. \& Tsuji K. (2013) The unusual neotenic system of the Asian dry wood termite, Neotermes koshunensis (Isoptera: Kalotermitidae). Sociobiology, 60, 65-68. doi: http://dx.doi.org/10.13102/sociobiology.v60i1.65-68

342 Miyata, H., Furuichi H. \& Kitade O. (2004) Patterns of neotenic differentiation in a subterranean termite, Reticulitermes speratus (Isoptera: Rhinotermitidae). Entomological Science, 7, 309-314. doi: 10.1111/j.1479-8298.2004.00078.x

345 Moore, B. (1974) Pheromones in the termite societies. Pheromones (ed M. Birch), pp. 250-266.

346 North-Holland Publishing, Amsterdam.

347 Myles, T.G. (1999) Review of secondary reproduction in termites (Insecta: Isoptera) with 348 comments on its role in termite ecology and social evolution. Sociobiology, 33, 1-43.

349 Noirot, C. 1990. Sexual castes and reproductive strategies in termites. Social Insects (ed W.

350 Engels), pp. 5-35. Springer, Berlin Heidelberg.

351 Page, R. E. \& Amdam G. V. (2007) The making of a social insect: developmental architectures 352 of social design. BioEssays, 29, 334-343. doi: 10.1002/bies.20549

353 Pichon, A., Kutnik M., Leniaud L., Darrouzet, E., Chaline, N., Dupont S. \& Bagnères A. (2007)

354 Development of experimentally orphaned termite worker colonies of two Reticulitermes species (Isoptera: Rhinotermitidae). Sociobiology, 50, 1015-1034.

356 Roisin, Y. (2000) Diversity and evolution of caste patterns. Termites: Evolution, Sociality, 
Roisin, Y. \& Korb J. (2011) Social organisation and the status of workers in termites. Biology of Termites: a Modern Synthesis. (eds D.E. Bignell, Y. Roisin \& N. Lo), pp. 133-164. Springer, Netherlands.

Su, N.-Y., Scheffrahn, R.H. \& Cabrera B.J. (2001) Native subterranean termites: Reticulitermes flavipes (Kollar), Reticulitermes virginicus (Banks), Reticulitermes hageni Banks (Insecta: Isoptera: Rhinotermitidae). University of Florida Cooperative Extension

Tian, L. \& X. Zhou. (2014) The soldiers in societies: defense, regulation, and evolution. International Journal of Biological Sciences, 10, 296-308. doi: 10.7150/ijbs.6847 Conserved class of queen pheromones stops social insect workers from reproducing.

371 Watanabe, D., Gotoh, H., Miura, T. \& Maekawa, K. (2014) Social interactions affecting caste development through physiological actions in termites. Frontiers in Physiology, 5, 127. doi: https://doi.org/10.3389/fphys.2014.00127

374 Watson, J. \& Abbey H.M. (1985) Development of neotenics in Mastotermes darwiniensis Froggatt: an alternative strategy. Caste Differentiation in Social Insects (eds J.A.L.

377 Watson, J.A.L., Metcalf E.C., \& Sewell J.J. (1975) Preliminary studies on the control of neotenic formation in Mastotermes Darwiniensis Froggatt (Isoptera). Insectes Sociaux, 22, 415426. doi: 10.1007/BF02224116 
380 Zhou, X., Oi F.M. \& Scharf M.E. (2006) Social exploitation of hexamerin: RNAi reveals a major caste-regulatory factor in termites. Proceedings of the National Academy of Sciences of

382 the United States of America, 103, 4499-4504. doi: 10.1073/pnas.0508866103

383 